\title{
Lentigo Maligna Melanoma
}

National Cancer Institute

\section{Source}

National Cancer Institute. Lentigo Maligna Melanoma. NCI Thesaurus. Code C9151.

A melanoma of the skin characterized by single cell infiltration of the papillary dermis by atypical melanocytes, in a background of lentigo maligna changes. 